\title{
Quaderni
}

QUADERNI Communication, technologies, pouvoir

69 | Printemps 2009

Universités sous influence du numérique et du management

\section{Le temps des « chercheurs-entrepreneurs » : sens et pouvoir du benchmarking dans l'« espace européen de la connaissance »}

"The age of the researcher-entrepreneur": the meaning and power of

benchmarking in the "European knowledge area"

Isabelle Bruno

\section{(2) OpenEdition}

Journals

Édition électronique

URL : http://journals.openedition.org/quaderni/321

DOI : 10.4000/quaderni.321

ISSN : 2105-2956

Éditeur

Les éditions de la Maison des sciences de l'Homme

Édition imprimée

Date de publication : 1 mai 2009

Pagination : 93-104

Référence électronique

Isabelle Bruno, "Le temps des « chercheurs-entrepreneurs » : sens et pouvoir du benchmarking dans I'« espace européen de la connaissance » », Quaderni [En ligne], 69 | Printemps 2009, mis en ligne le 05 avril 2012, consulté le 20 avril 2019. URL : http://journals.openedition.org/quaderni/321 ; DOI :

10.4000/quaderni.321 


\section{$D$ ossier}

\section{le temps des}

\section{"chercheurs-}

\section{entrepreneurs" :}

sens et pouvoir

\section{du benchmarking}

dans l'"espace européen de la connaissance"

\section{Isabelle Bruno}

Maître de conférences en science politique CERAPS - Université Lille 2
«Oubliée l'époque où universités et entreprises se regardaient en chiens de faïence... En quelques années, une nouvelle organisation de la recherche s'est mise en place autour de la figure emblématique du chercheur-entrepreneur $\gg{ }^{1}$. C'est en ces termes que le magazine d'information sur la recherche européenne, publié par l'unité Information et Communication de la direction générale (DG) Recherche de la Commission européenne, présente la « révolution culturelle » à l'œuvre dans les domaines scientifique et technologique. Fers de lance d'une " économie fondée sur la connaissance », ceux qu'on appelait autrefois les savants auraient désormais vocation non seulement à générer de nouveaux savoirs, mais surtout à les convertir en innovations brevetables et commercialisables. Cette métamorphose serait rendue nécessaire par le contexte de globalisation marchande. «Le temps où, traditionnellement, les savoirs acquis dans l'espace scientifique académique constituaient un patrimoine ouvert, mis à la disposition de tous, appartient au passé. Dans le champ des connaissances, production rime aujourd'hui avec protection et exploitation. [...] Or cette exploitation, dans une économie de marché, a une dimension intrinsèquement économique $\gg{ }^{2}$. Donc - poursuivons le syllogisme l'activité consistant à produire des connaissances s'inscrit pareillement dans une logique économique. Il est ainsi suggéré que le chercheur est tenu, « par la force des choses », d'observer les lois du marché et l'exigence corrélative de compétitivité. L'interventionnisme gouvernemental qui, en transformant les conditions d'exercice de son métier et le statut attaché à son rôle social, le conduit à agir comme un entrepreneur, est passé sous silence conformément au mythe de la « main invisible ». 
Loin de procéder par génération spontanée, l'avènement des « chercheurs-entrepreneurs » n'est pas une donnée naturelle, ni même un fait historique, mais une visée politique. Il est censé découler de la construction d'un « espace européen de la recherche » (EER), l'un des principaux chantiers ouverts par la stratégie de Lisbonne lancée en $2000^{3}$. Afin que l'Union devienne en une décennie "l'économie de la connaissance la plus compétitive et la plus dynamique du monde $»^{4}$, les chefs d'État et de gouvernement réunis en sommet extraordinaire ont alors approuvé le projet d'EER porté par la Commission. Celle-ci est partie du constat que l'insuffisance et l'inefficacité des investissements européens dans la connaissance s'expliquaient par la «fragmentation des efforts, l'isolement et le cloisonnement des systèmes nationaux de recherche, la disparité des régimes réglementaires et administratifs $»^{5}$. Pour y remédier, elle a proposé de créer un «marché européen d'offre et de demande de connaissances et de technologies », nécessitant pour son développement et son fonctionnement «la définition d'une vraie politique européenne de recherche $»^{6}$. Non pas une politique communautaire ayant pour instrument le droit supranational, mais plutôt un dispositif coordonnant les actions gouvernementales en vue d'une fin commune : l'édification d'un « marché des connaissances ». Le Conseil européen de Lisbonne a ainsi encouragé « l'élaboration d'une méthode ouverte de coordination [MOC] destinée à évaluer les performances des politiques nationales de recherche et de développement $\gg{ }^{7}$. Par là, il entend une démarche de quantification et d'évaluation comparative (benchmarking) en quatre temps qui « consiste à :

- définir des lignes directrices pour l’Union, assorties de calendriers spécifiques pour réaliser les objectifs à court, moyen et long terme fixés par les États membres ;

- établir, le cas échéant, des indicateurs quantitatifs et qualitatifs et des critères d'évaluation par rapport aux meilleures performances mondiales, qui soient adaptés aux besoins des différents États membres et des divers secteurs, de manière à pouvoir comparer les meilleures pratiques ;

- traduire ces lignes directrices européennes en politiques nationales et régionales en fixant des objectifs spécifiques et en adoptant des mesures qui tiennent compte des diversités nationales et régionales ;

- procéder périodiquement à un suivi, une évaluation et un examen par les pairs, ce qui permettra à chacun d'en tirer des enseignements $»^{8}$.

Cet article ne s'inscrit pas dans les controverses qui épuisent le sujet en l'enfermant dans l'alternative suivante : soit la MOC est conçue comme une méthode «molle » vouée à se durcir en se coulant dans le moule classique d'intégration par le droit ; soit elle est envisagée comme support d'une « nouvelle gouvernance » jugée souhaitable car fondée en rationalité, mais dont la postérité serait mise en péril par les conservatismes souverains et l'impuissance de la raison gestionnaire à les vaincre 9 . Pour sortir de cette impasse normative, notre thèse déplace l'attention sur un objet technique - le benchmarking - dépolitisé en raison de la neutralité qui est a priori attachée à son usage, et jugé de ce fait moins noble que les textes juridiques, les négociations diplomatiques ou les batailles institutionnelles concentrant l'intérêt des européanistes. Afin de lui restituer sa portée éminemment politique, il l'aborde suivant une approche généalogique qui va à rebours des 
théories managériales postulant son utilité universelle. Son propos n'est pas d'estimer le degré d'efficacité d'un outil au regard des objectifs qui lui ont été assignés dans le cadre de la stratégie de Lisbonne. Il vise plus fondamentalement à déchiffrer les effets de codification et de prescription produits par le benchmarking, c'est-àdire la façon dont il renseigne ses praticiens sur ce qui est à savoir et sur ce qui est à faire. Par la mise en nombre et la mise en comparaison des résultats nationaux, ce procédé de coordination interétatique objective des différentiels de performance et les rend visibles dans des palmarès qui réordonnent « grands » et « petits » pays à l'aune de leur grandeur compétitive. Il borne ainsi le champ des possibles en délimitant ce qui est faisable par la mesure de ce qui a été fait, le souhaitable se réduisant alors aux meilleurs scores enregistrés.

En mobilisant les conclusions d'une enquête doctorale menée entre 2001 et $2006^{10}$, nous interrogerons d'une part le sens que cette technologie de gouvernement imprime au dessein d'une « Europe de la connaissance » et d'autre part le « modèle gestionnaire d'exercice du pouvoir $»^{11}$ qu'elle matérialise. Après avoir pisté son cheminement depuis l'industrie japonaise jusqu'à l'Union européenne, en passant par les relais du New Public Management, nous tâcherons de caractériser la « discipline indéfinie » qui fait la force de cette méthode faible. Cet article souhaite en cela apporter quelques éléments de réflexion pour saisir comment le benchmarking rend la concurrence opératoire comme principe d'organisation sociale, et tend ainsi à soumettre toutes les activités humaines, notamment scientifiques et éducatives, à un même impératif de compétitivité.

\section{Dans l'entre-deux de la coopération et de la compétition : le «bon sens» managérial du benchmarking}

Qu'est-ce que le benchmarking? Au premier abord, cette dénomination barbare prête volontiers à la raillerie, surtout parmi les francophones enclins à une certaine défiance à l'égard des anglicismes. Elle évoque un gadget à la mode qui compose, avec le downsizing, l'outsourcing ou le reengineering, la « panoplie du bon manager $»^{12}$. Autant de termes saugrenus dont le sérieux laisse sceptique mais qui, en se propageant dans le langage courant, acquièrent la force de l'évidence. Bien que le benchmarking tende à se banaliser depuis une décennie, il n'en demeure pas moins un objet énigmatique. Le mot est devenu familier, mais le flou qui entoure la chose ne s'est toujours pas dissipé. Les Français le traduisent par « étalonnage des performances » ou « évaluation comparative »; les Québécois préférant parler de « parangonnage ». Quelle que soit l'appellation retenue, ce procédé de gestion par objectifs consiste à repérer un étalon ou un parangon, c'està-dire un modèle avec qui se comparer dans le but de combler l'écart de performance qui vous en sépare. La prolifération actuelle de ses usages dans toutes les formes d'organisation, quelle que soit leur fonction, tend à le naturaliser, à l'inscrire dans le sens commun comme la réponse idoine au besoin présumé universel de compétitivité. Dès lors que son principe d'ordonnancement social est reconnu utile, sa pratique ne soulève plus que des problèmes ponctuels de méthode, à résoudre au cas par cas, sans que sa finalité et son bien-fondé soient mis en question. À rebours des approches utilitariste ou fonctionnaliste, il nous faut interroger la rationalité managériale 
qui rend cette technique nécessaire au «bon gouvernement » des hommes et de leurs institutions. C'est en l'historicisant que nous pouvons lui restituer sa contingence et sa singularité contre une scientificité neutralisante.

"Qui veut s'améliorer doit se mesurer, qui veut être le meilleur doit se comparer $»$ : telle est la devise de Robert Camp, qui a dirigé chez Rank Xerox le premier programme de benchmarking lancé aux États-Unis en 1979 et s'en est fait le théoricien dans un ouvrage à succès ${ }^{13}$. Ce mot d'ordre s'inspire directement du mouvement Kaizen, impulsé par Toyota dans les années 1950 puis généralisé à l'ensemble des firmes japonaises, qui fait prévaloir l'« amélioration continue » et la « qualité totale » contre les efforts mimétiques et productivistes. Dans un ouvrage qui prend pour titre un précepte du toyotisme - « penser à l'envers »-, Benjamin Coriat a montré en quoi ce système de production rompt avec «l'héritage venu de l'Occident ». Son originalité provient de ce qu'il dépasse la contradiction théorique instaurée entre les relations de concurrence et de collaboration, et qu'il aménage les conditions de leur coexistence pratique. C'est en jouant de leur antagonisme qu'il ajuste un dispositif dans l'entre-deux du marché et de l'organisation, des sciences économique et managériale. La généralisation de cette approche explique le «dosage savant de coopération et de mise en compétition dont est constitué le rapport inter-firmes dans la société japonaise $»^{14}$. Si l'industrie japonaise est parvenue à s'engager sur un «nouveau chemin de compétitivité », ce n'est donc pas simplement en abandonnant la règle d'or du « copier fait gagner ». Il lui a fallu sortir des sentiers battus par les Occidentaux, et substituer au raisonnement autoréférentiel qu'expriment les slogans «one best way » ou « NIH » (Not Invented Here), une logique différentielle qui commande de «s'ouvrir aux meilleures pratiques possibles ».

C'est en cela que la littérature managériale voit dans le benchmarking le fer de lance d'une « révolution culturelle » : transformer la « culture d'entreprise » par une démarche comparative qui « implique d'être assez modeste pour admettre que quelqu'un d'autre est meilleur dans un domaine, et assez sage pour essayer d'apprendre comment l'égaler et même le surpasser $»^{15}$. À partir des années 1990, un foisonnement de publications - guides, manuels, revues spécialisées - contribue à en rationaliser la pratique. Leurs auteurs normalisent la procédure itérative mise au point par Camp, qui compte dix étapes regroupées en quatre phases (planification; analyse ; intégration ; action) ${ }^{16}$; ils l'objectivent dans des textes à prétention scientifique et l'illustrent par d'innombrables études de cas. Autrement dit, ils disciplinent cette technique de « collaboration compétitive ». Friands de buzzwords, le management étasunien a même fait de cet oxymore un mot-valise : «co-opetition $»^{17}$. Il désigne par là un agencement organisationnel « révolutionnaire » en ce qu'il combine les principes apparemment antagoniques de co-operation et de competition. Un modus vivendi aurait ainsi été trouvé entre le dogme néoclassique selon lequel l'allocation des ressources est optimale en condition concurrentielle, et le besoin de coordination suscité par les imperfections du marché. En dénouant cette contradiction, le benchmarking aménage les conditions de possibilité - dans l'ordre des idées et dans l'ordre de l'action - de l'utopie néolibérale. 
L'analogie communément admise entre la gestion d'une entreprise et l'administration d'un État inscrit en toute cohérence ce procédé dans la mouvance du New Public Management (NPM). Sans entrer dans le détail de ce courant composite, l'action politique par laquelle il entend «réinventer le gouvernement $\|^{18}$ ne peut être éludée. Les « nouveaux gestionnaires publics » exercent un pouvoir de conviction et de ralliement à tous les échelons des appareils étatiques. Certes, leur agenda réformiste et leur ambition déclarée de renverser la frontière prétendue inébranlable bien qu'ayant toujours été poreuse - entre sphères publique et privée, entre terrains politiques et économiques, n'ont rien d'inédit. Ils s'inscrivent dans le prolongement d'une tradition administrative qui ne s'est jamais interdit de recourir à la « rationalité calculatrice » et aux techniques marchandes pour gérer l'espace réputé souverain de l'État ${ }^{19}$. Toutefois, il serait réducteur de dénier toute spécificité au changement étiqueté « NPM », et de limiter son envergure à un effet collatéral du "consensus de Washington ${ }^{20}$ qui, depuis les années 1980, préside au tournant néolibéral pris par tous les pays industrialisés. La mutation, plus radicale, touche à la fois aux manières de penser et d'agir qui régissent les pratiques gouvernementales. Les promoteurs du NPM véhiculent non seulement l'idéal d'un «Étatstratège » devenu dominant dans les années $1990^{21}$, mais aussi l'ingénierie administrative, la boîte à outils qui permet à ses agents de le réaliser. Ils rendent opérationnelle une façon managériale de gouverner à distance et la systématise dans un régime singulier de gouvernementalité, plus connu sous le nom de «gouvernance».

Avec la « bonne gouvernance » comme cheval de bataille et le benchmarking comme arme de prédilection, l'Organisation de Coopération et de Développement Économiques (OCDE) fait figure de tête de pont de la « nouvelle gestion publique » dans tous ses pays membres ${ }^{22}$. Depuis 1990, la Direction de la gouvernance publique et du développement territorial s'appuie sur son réseau PUMA (PUblic MAnagement) pour en diffuser les principes d'action sous le sceau d'une expertise légitime. Par la publication de données comparatives et leur examen collégial en comité, elle familiarise les hauts fonctionnaires avec l'étalonnage des performances comme pivot d'une « gestion axée sur les résultats ». Dès 1994, une étude expose clairement la « révolution culturelle» dont ce procédé serait le ferment, en faisant passer l'administration «d'une culture d'application des règles à une culture de la performance $\gg{ }^{23}$. En 1996, la réunion des participants au sous-réseau du PUMA sur la gestion des performances (Performance Management Network) donne lieu à un rapport sur les pratiques de benchmarking dans le secteur public ${ }^{24}$.

La même année et dans le même esprit, la Commission européenne organise en collaboration avec la Table ronde des industriels européens (ERT) - club élitiste réunissant une quarantaine de capitaines d'industrie parmi les plus puissants d'Europe - un séminaire sur le thème « Le benchmarking pour les responsables politiques : vers la compétitivité, la croissance et la création d'emplois $»^{25}$. Elle ne se contente pas de le promouvoir auprès des entreprises européennes, afin de conforter leur aptitude à conquérir des parts de marché au niveau mondial ${ }^{26}$. À l'instar de l'OCDE, elle vise les gouvernants nationaux comme cible privilégiée. Dans une communi- 
cation de 1997 intitulée «Benchmarking : mise en ouvre d'un instrument destiné aux acteurs économiques et aux autorités publiques », elle les encourage à utiliser cette technique managériale pour administrer efficacement leur population et leur territoire ${ }^{27}$. À cet effet, un «groupe de haut niveau sur le benchmarking » est créé par la DG III (Industrie) avec l'intention d'en accréditer le bien-fondé. Dans le rapport qu'il remet à la Commission en 1999, il préconise un étalonnage systématique des « conditions cadres » de l'activité économique afin que les États membres s'efforcent de les rendre plus attractives aux yeux des investisseurs et des travailleurs qualifiés ${ }^{28}$. L'introduction du benchmarking dans les rouages communautaires et interétatiques apparaît tellement incontournable que Jacques Santer, alors président de la Commission, a pu affirmer sur un ton d'évidence : "We are all benchmarkers now! ${ }^{29}$. Dès l'année suivante, le Conseil européen de Lisbonne lui donne effectivement raison en consacrant le recours au benchmarking comme technique de coordination intergouvernementale.

\section{La quête indéfinie d'une performance compétitive : le pouvoir disciplinaire du benchmarking}

Avec la «co-opétition », le management a façonné un principe associatif plus souple que les règles formelles du droit et plus pragmatique que la concurrence pure de l'économie. Quoiqu'elle ne reprenne pas le mot, la MOC pratique la chose. Elle s'inspire de ce savoir-faire pour agencer une coordination intergouvernementale qui procède par la planification d'objectifs et la mesure d'écarts, écarts que les gouvernants doivent s'efforcer de combler pour prouver la compétitivité de leur pays. Une telle mise à l'épreuve les incline à s'aligner sur la « conduite économique de l'entrepreneur moderne» agissant «conformément à un plan, en vue d'une fin et sur la base du calcul $»^{30}$. Au moyen du benchmarking, la MOC consiste ainsi à arranger un « dispositif collectif de calcul» qui met en rapport les États, leur population et leur territoire, comme le marketing met en balance des produits, suivant un «processus de classification, de groupement et d'appariement » les rendant « $\grave{a}$ la fois comparables et différents $»{ }^{31}$. L'enjeu de l'étalonnage interétatique est de faire prévaloir un principe d'équivalence contre l'incommensurabilité des entités souveraines. En équipant les membres de l'Union d'un même arsenal d'outils statistiques et classificateurs, il les déplace d'un champ de négociation internationale, traditionnellement régi par le droit, vers un terrain de compétition où leurs scores sont enregistrés et confrontés à l'aune d'indicateurs communs de performance. C'est là que se déploie l'EER, au rythme des cycles de benchmarking qui ambitionnent de remédier - par la « collaboration compétitive » de tous ses acteurs - au cloisonnement entre secteurs public et privé, à la fragmentation des activités de recherche et à la dispersion des efforts de financement. L'européanisation des systèmes nationaux de recherche ne signifie donc ni le retrait des États supplantés par les instances suprationales, ni la mise en congé des pouvoirs publics par les forces privées. Elle manifeste plutôt une reconfiguration des niveaux décisionnels et opérationnels de l'action politique, des modes administratifs d'intervention et de contrôle à distance qui ne perdent rien en intensité mais se donnent de nouvelles cibles (autres finalités sociales, autres 
catégories bénéficiaires) et de nouveaux moyens (moins directs, plus incitatifs).

La perspective d'une « Europe compétitive » projetée par la stratégie de Lisbonne est asymptotique : elle figure un idéal directeur qui oriente les conduites. La ligne magistrale qu'elle trace n'est pas pour autant évanescente dans le ciel des idées. Elle se matérialise dans un programme d'actions que vient cimenter le dispositif de benchmarking avec ses «objectifs quantifiés », ses "chiffres clés », ses «tableaux de bord», ses « rapports d'évaluation », ses « indicateurs structurels», ses bases de données statistiques et leur «profil de qualité ». Tous ces maillons se nouent les uns avec les autres et durcissent l'exigence de la compétitivité en y faisant constamment référence. En rendant cette exigence tangible, quantifiable, calculable, le benchmarking plie les gouvernants à une « discipline indéfinie ». Pourquoi « indéfinie » ? Parce que la norme de compétitivité est endogène à la course sans fin à laquelle cette technique livre ses praticiens. Le benchmark, c'est-à-dire la cible qu'il leur assigne comme référence, est idéalement fugitif : il n'est fixé que pour être rejoint sinon dépassé, et laisser ainsi la place au nouvel étalon arrivé en tête. De fait, il est inaccessible. Poser la compétitivité comme un but à atteindre au moyen du benchmarking, qui consiste à chiffrer des écarts de performance, c'est objectiver une distance que l'opération même de sa réduction reproduit indéfiniment. L'expression de « discipline indéfinie » est empruntée à Michel Foucault, qui l'emploie dans un autre contexte pour désigner « une procédure qui serait à la fois la mesure permanente d'un écart par rapport à une norme inaccessible et le mouvement asymptotique qui contraint à la rejoindre à l'infini $»^{32}$.
La pratique du benchmarking n'emprisonne pas pour autant les gouvernants dans une « cage d'acier». Elle participe d'une «technologie d'engagement ${ }^{33}$ qui les place en condition de rationaliser leurs conduites dans un but partagé, tout en les laissant libres d'agir à leur convenance. C'est cette liberté d'action qui produit des performances et des distinctions. Le benchmarking discipline l'acte de gouverner en ce qu'il incite les gouvernants à faire aussi bien, sinon mieux, que leurs pairs; à se positionner en tête des classements, ou tout du moins à ne pas afficher une contre-performance. Pour ce faire, il les renseigne par un flux d'informations chiffrées, régulièrement mises à jour, et il les familiarise avec un mode gestionnaire de délibération et de prise de décision qui procède par quantification et par comparaison. En acceptant d'étalonner leurs performances nationales, les États membres se sont autoprescrit certaines règles : rendre compte annuellement de leurs initiatives dans des plans d'action nationaux; mesurer leurs résultats au regard d'indicateurs statistiques communs; se fixer eux-mêmes des objectifs quantitatifs qui serviront à leur évaluation; se faire réciproquement superviseurs de leurs pairs. Ce faisant, ils se contrôlent à distance avec le soutien logistique de la Commission (et de son office statistique Eurostat) qui se contente d'équiper cette surveillance multilatérale, au moyen notamment des rapports conjoints, des listes d'indicateurs, des publications qui rendent visibles les benchmarks et les échéanciers, etc. Tous ces éléments contribuent à affermir la croyance en une compétitivité de l'Union qui pousse ses «champions » à faire front commun avec les « retardataires » dans un espace mondialisé de concurrence. 
Dénué de toute force contraignante, le benchmarking ne braque pas les autorités gouvernementales car, en apparence, il ne met en péril ni leur autonomie, ni leur pouvoir de décision. Il est simplement censé servir d'aiguillon, en stimulant leur volonté de progresser vers les objectifs convenus avec leurs homologues. Il est en cela parfaitement compatible avec le principe de subsidiarité ${ }^{34}$ et le respect des idiosyncrasies nationales. C'est pourquoi on le destine aux domaines d'action publique qui constituent les bastions de la souveraineté étatique. Pour autant, il ne les investit pas sans y accomplir sa révolution silencieuse. Qu'il mette en rapport les pays membres à travers leurs taux d'emploi ou d'investissement dans la $\mathrm{R} \& \mathrm{D}$, le benchmarking crée du lien en insufflant un esprit de groupe, sinon d'entreprise au sein de l'Union. Il alimente une émulation en quantifiant et en classant les performances de chacun au regard du but visé par tous $^{35}$; et il produit une cohésion d'ensemble en les listant dans des tableaux croisés. Ces grilles de benchmarking mettent en présence les individualités souveraines en les objectivant dans des chiffres comparables. Autrement dit, elles sapent le caractère d'incommensurabilité inhérent aux relations interétatiques et les déplacent sur un plan d'équivalence qui autorise leur confrontation. La médiation du benchmarking transforme ainsi les rapports de force - propres aux relations entre États souverains - en rapports de raison, suivant une méthode gestionnaire qui animent leur coopération d'une visée compétitive.

\section{Conclusion}

L'acte d'étalonner les organisations sociales

- qu'il s'agisse d'États, d'universités ou de laboratoires de recherche - est proprement politique, en ce qu'il les réordonne selon une certaine hiérarchie de valeurs. Il les passe au crible de grilles d'indicateurs chiffrés qui sont censés évaluer leurs performances, non pas dans l'absolu ni dans le temps, mais toujours relativement aux « autres », les « pairs » faisant à cet égard davantage figure de concurrents que d'homologues ou d'égaux. Il les redistribue le long d'une échelle classificatrice qui valorise non pas leur puissance militaire, leur vertu éducative ou leur excellence scientifique mais leur compétitivité. Or cette grandeur relative n'est pas un donné. Elle traduit leur capacité à afficher le meilleur score, du moins à faire mieux que les autres, dans une compétition qui ne préexiste pas au palmarès puisqu'elle est paramétrée par les critères du classement lui-même. Elle est donc le produit d'une chaîne socio-technique qui en construit la mesure. Et le benchmarking n'en est pas un simple maillon instrumental. Il est la médiation qui imprime à cette chaîne son sens axiologique et sa charge normative. Sa force cohésive procède de logiques de différenciation, et non pas d'uniformisation. Il associe ses objets en les confrontant et en les distinguant ; il les met en présence sur un terrain chiffré et les engage dans une même compétition. Ce faisant, il tend à généraliser à l'ensemble des relations sociales le «principe d'association dissociatif» propre au lien économique ${ }^{36}$, et actualise ainsi l'idée d' « économie sociale de marché » forgée par les ordolibéraux dans l'Europe d'aprèsguerre $^{37}$. Le principe régulateur du marché, que le benchmarking rend opératoire dans les domaines non marchands, désigne non pas l'équivalence de l'échange mais l'inégalité concurrentielle, non pas l'uniformité et l'homogénéisation 
de la marchandise mais la «multiplicité et la différenciation des entreprises $»^{38}$. La matrice réglée sur les « mécanismes de la concurrence » engendre ainsi une "société d'entreprise » qui ne se confond pas avec une «société de supermarché $»^{39}$. Y règne l'entrepreneur qui produit et innove pour se distinguer, qui prend des risques en investissant ses capitaux (financiers comme humains) et qui compte sur l'État pour sécuriser son environnement. Voilà l'homme nouveau auquel sont censés se conformer les chercheurs, les enseignants et leurs étudiants, en tant qu'acteurs de l' « économie de la connaissance » et en vue d'une « Europe compétitive».
$\mathrm{N} \cdot \mathrm{O} \cdot \mathrm{T} \cdot \mathrm{E} \cdot \mathrm{S}$

1. Commission européenne, « Le temps des chercheurs-entrepreneurs », in RDT info, $\mathrm{n}^{\circ} 35$, Bruxelles, octobre 2002, p. 6 .

2. Commission européenne, «Vers un marché des connaissances », in RDT info, $\mathrm{n}^{\circ} 34$, Bruxelles, juillet 2002, p. 16.

3. Notons que, depuis 2005 , cette stratégie a été recentrée sur trois priorités ( « Investir davantage dans la connaissance et l'innovation »; « Exploiter le potentiel des entreprises, en particulier les PME »; «Investir dans le capital humain et moderniser le marché du travail »), et traite non seulement la recherche et l'innovation mais aussi l'éducation («triangle de la connaissance »). Il s'ensuit qu'il est devenu courant de parler d'un « espace européen de la recherche et de l'enseignement supérieur », concernant au premier chef les universités. Dans le souci de « se doter d'une économie réellement moderne et compétitive », le Conseil européen de mars 2008 les a ainsi appelées à « développer des partenariats avec le monde des entreprises » et à « accroître la mobilité transfrontière » de leurs scientifiques, enseignants et étudiants (in Conseil européen, "Conclusions de la Présidence ", Sommet européen de Bruxelles, 13-14 mars 2000, Nr : 7652/08, §8).

4. Conseil européen, « Conclusions de la Présidence », Sommet européen de Lisbonne, 23-24 mars 2000, Nr : $100 / 1 / 00, \S 5$.

5. Commission européenne, «Vers un espace européen de la recherche », Communication de la Commission au Conseil, au Parlement européen, au Comité économique et social et au Comité des régions, COM (2000) 6 final, p. 7.

6. Ibid., p. 8.

7. Conseil européen, op. cit. , $\$ 13$. 
8. Ibid., $\$ 37$.

9. Voir, entre autres, Adrienne Héritier, « New Modes of Governance in Europe: Policy-Making without Legislating ? », in Adrienne Héritier (ed.), Common Goods. Reinventing Europe and International Governance, Rowman \& Littlefield, Lanham, 2002, pp. 185-206 ; Joanne Scott, David Trubek, « Mind the Gap : Law and New Approaches to Governance in the European Union », in European Law Journal, vol. 8, $\mathrm{n}^{\circ}$ 1, Oxford, 2002, p. 1-18; Renaud Dehousse, « La méthode ouverte de coordination : quand l'instrument tient lieu de politique », in Pierre Lascoumes, Patrick Le Galès (dir.), Gouverner par les instruments, Presses de Sciences po, Paris, 2004, pp. 331-356.

10. Isabelle Bruno, «Déchiffrer «l'Europe compétitive». Étude du benchmarking comme technique de coordination intergouvernementale dans le cadre de la stratégie de Lisbonne ", Thèse de doctorat en science politique, Institut d'Études Politiques de Paris, 2006 (consultable en ligne sur le site du Réseau Européen d'Analyse des Sociétés Politiques $<$ http://www.fasopo.org/reasopo.htm\#jr >). 11. Albert Ogien, L'esprit gestionnaire. Une analyse de l'air du temps, Paris, Éditions de l'EHESS, 1995. Sur la propagation de ce modèle en terme de «gouvernance», lire du même auteur: «La gouvernance, ou le mépris du politique », in Cités, $n^{\circ}$ 32, Paris, 2007, pp. 137-155.

12. D'après le titre d'un article d'Enjeux les Échos (mai 2003, p. 86-88) qui publie le «palmarès des outils les plus utilisés » par les entreprises, classant le benchmarking au deuxième rang derrière la planification stratégique.

13. Robert Camp, Benchmarking : The Search for Industry Best Practices that Lead to Superior Performance, Milwaukee, Quality Press, 1989 (traduit en français sous le titre Le benchmarking: pour atteindre l'excellence et dépasser vos concurrents,
Paris, Les éditions d'organisation, 1992).

14. Benjamin Coriat, Penser à l'envers. Travail et organisation dans l'entreprise japonaise, Paris, Christian Bourgois, 1991, p. 124.

15. Jean Brilman, Les meilleures pratiques de management, Paris, Les éditions d'organisation, 2003, p. 288.

16. La MOC, telle qu'elle est décrite dans les conclusions de Lisbonne, reprend ces quatre temps (cf. supra).

17. Adam Brandenburger, Barry Nalebuff, Coopetition: A revolutionary mindset that combines competition and co-operation, New York, Currency Doubleday, 1998

18. D'après le titre d'un ouvrage célèbre aux ÉtatsUnis, auquel l'administration Clinton-Gore se référa pour réformer la gestion publique: Ted Gaebler, David Osborne, Reinventing Goverment, AddisonWesley, Reading, 1992.

19. Sur le "rôle décisif joué par le développement du commerce, à la fin du Moyen Âge, dans la transformation des manières de penser 》 et des pratiques politiques, voir Michel Senellart, Les arts de gouverner. Du regime médiéval au concept de gouvernement, Paris, Seuil, 1995. Sur la maxime "administration is business » et les autres emprunts du NPM au «management scientifique» et à l'« idéologie du marché », voir Ezra Suleiman, Dismantling Democratic States, Princeton, Princeton University Press, 2003.

20. Yves Dezalay, Bryant Garth, «Le 'Washington consensus': contribution à une sociologie de l'hégémonie du néolibéralisme», Actes de la recherche en sciences sociales, 121-122, Paris, mars 1998, pp. 3-23.

21. Philippe Bezes, «Le modèle de «l'Étatstratège» : genèse d'une forme organisationnelle dans l'administration française », in Sociologie du travail, 
47, Paris, 2005, pp. 431-450.

22. Jean-Michel Saussois, « Partird'une 'commande' : analyser l'action diffusionniste de l'OCDE dans ses pratiques de benchmarking et de propagation sur les nouvelles pratiques en matière de management public », Contribution au VIIe Congrès de l'AFSP, Lille, septembre 2002.

23. OCDE, «La gestion des performances dans l'administration : mesure des performances et gestion axée sur les résultats », Études hors série sur la gestion publique, $\mathrm{n}^{\circ}$ 3, Paris, OCDE, 1994.

24. OECD, « Benchmarking, evaluation and strategic management in the public sector ", Papers presented at the 1996 Meeting of the Performance Management Network of the OECD's Public Management Service, OCDE/GD (97) 50, Paris.

25. Préparé par le groupe de travail « Compétitivité » de l'ERT, ce séminaire a réuni le 21 mars 1996 à Bruxelles plus de 80 représentants des États membres, des institutions communautaires et du secteur industriel (ERT, « Benchmarking for PolicyMakers : The Way to Competitiveness, Growth and Job Creation ", Report based on the findings of a seminar organized by the European Commission and the ERT (21 March 1996), October 1996).

26. European Commission, Benchmarking the Competitiveness of European Industry, COM (96) 463 final, 9 October 1996.

27. European Commission, Benchmarking: Implementation of an Instrument Available to Economic Actors and Public Authorities, Com (97) 153/2, 16 April 1997.

28. High Level Group on Benchmarking (European Commission/DG III), «First Report by the High Level Group on Benchmarking », Benchmarking Papers, $\mathrm{n}^{\circ}$ 2, 1999.

29. Cité dans Keith Richardson, «Big Business and the European Agenda», Working Paper in
Contemporary European Studies, 35, University of Sussex, Sussex European Institute, September 2000, p. 26.

30. Werner Sombart, Le bourgeois. Contribution à l'histoire morale et intellectuelle de l'homme économique moderne, Payot, Paris, 1966, p. 145.

31. Michel Callon, Fabian Muniesa, «Les marchés économiques comme dispositifs collectifs de calcul», in Réseaux, $\mathrm{n}^{\circ}$ 122, Issy-les-Moulineaux, 2003, pp. 189-233, p. 204.

32. Michel Foucault, Surveiller et punir. Naissance de la prison, Paris, Gallimard/Tel, 1975, p. 264.

33. Jens Henrik Haahr, «Open co-ordination as advanced liberal government», in Journal of European Public Policy, vol.11, $\mathrm{n}^{\circ} 2$, London, April 2004, pp. 209-230

34. "Dans les domaines qui ne relèvent pas de sa compétence, la Communauté n'intervient, conformément au principe de subsidiarité, que si et dans la mesure où les objectifs de l'action envisagée ne peuvent pas être réalisés de manière suffisante par les États membres et peuvent donc, en raison des dimensions ou des effets de l'action envisagée, être mieux réalisés au niveau communautaire » (extrait de l'article 5 du traité instituant la Communauté européenne).

35. À savoir une moyenne européenne de 70\% de taux d'emploi ( $60 \%$ pour les femmes) et de $3 \%$ d'intensité de R\&D (part des Dépenses Intérieures de Recherche et Développement dans le Produit Intérieur Brut) en 2010. Sur ces deux exemples, voir respectivement Gilles Raveaud, «Au cœur de la stratégie européenne pour l'emploi: le taux d'emploi», Éducation et sociétés, ${ }^{\circ} 18$, Paris, 2006, p. 17-33; et Isabelle Bruno, À vos marques ${ }^{\circledR}$, prêts... cherchez! La stratégie européenne de Lisbonne, vers un marché de la recherche, Bellecombe-en-Bauges, Éditions du Croquant, 2008. 
36. Michel Foucault, Naissance de la biopolitique. Cours au Collège de France (1978-1979), Paris, Gallimard/Seuil, 2004, p. 310.

37. Ibid., p. 150.

38. Ibid. , p. 155.

39. Ibid., p. 152.
$R \cdot E \cdot S \cdot U \cdot M \cdot E$

À rebours des approches postulant la neutralité des techniques managériales quant aux fins poursuivies, cet article se propose d'étudier les effets politiques produits par l'usage du benchmarking comme outil de construction d'un «Espace Européen de la Recherche ». Il interroge d'une part le sens que cette technologie de gouvernement imprime au dessein d'une « Europe de la connaissance », et d'autre part le pouvoir disciplinaire qu'elle exerce au moyen d'un dispositif de gestion par objectifs et d'évaluation comparative des performances. En exposant les résultats d'une enquête généalogique, cet article souhaite apporter quelques éléments de réflexion pour saisir comment le benchmarking rend la concurrence opératoire comme principe d'organisation sociale, et tend ainsi à soumettre toutes les activités humaines - notamment scientifiques et éducatives - à un même impératif de compétitivité.

"The age of the researcher-entrepreneur" : the meaning and power of benchmarking in the "European knowledge area"

Against studies assuming that management techniques are toothless regarding ends, this article intends to emphasize the political effects produced by the use of benchmarking as a construction tool to build the « European Research Area ». It examines, on the one hand, the meaning that this technology of government gives to the design of a «knowledge-based Europe » and, on the other hand, the disciplinary power it exerts through management by objectives and performance peer review. Working on the results of a genealogical investigation, this article suggests some new avenues to understand how benchmarking makes competition effective as a principle of social organization, and thereby tends to subject all human activities - especially scientific and educative ones - to the same competitiveness imperative. 